\title{
Preliminary Assessment of Safety and Immunogenicity of an Inactivated Whole-Virion Vaccine against Influenza A (H1N1) Pdm09 Containing Aluminum Hydroxide Adjuvant: A Randomized, Blinded Phase I Clinical Study
}

Kaissar Tabynov ${ }^{1 *}$, Berik Khairullin ${ }^{1}$, Zhailaubay Kydyrbayev $^{1}$, Nurlan Sandybayev $^{1}$, Marina Stukova ${ }^{2}$, Marianna Erofeeva², Anna Polina Shurygina ${ }^{2}$, Oleg Kiselev ${ }^{2}$, Seidigapbar Mamadaliev ${ }^{1}$ and Abylai Sansyzbay ${ }^{1}$

${ }^{1}$ Research Institute for Biological Safety Problems (RIBSP), Gvardeiskiy, Kazakhstan

${ }^{2}$ Research Institute of Influenza, St. Petersburg, Russian Federation, Russia

\begin{abstract}
We performed a randomized, blinded, dose-dependent placebo-controlled phase I clinical study of single administration of Refluvac ${ }^{\circledR}$, a monovalent inactivated whole-virion vaccine against pandemic influenza A (H1N1) pdm09 containing aluminum adjuvant, in healthy volunteers aged 18-60. Single intramuscular injection at doses of 3.75, 7.5, or $15.0 \mu \mathrm{g}$ hemagglutinin (HA) identified no safety issues in adult volunteers ( $n=12$ per group); no severe or serious vaccination-related adverse events were observed. Only mild/moderate local adverse events $(n=3 / 12,25 \%$ in $7.5 \mu \mathrm{g}$ HA arm, ) and one moderate systemic reaction ( $n=1 / 12,8.3 \%$ in $15.0 \mu \mathrm{g} \mathrm{HA}$ arm) were observed. In volunteers vaccinated at $3.75 \mu \mathrm{g} \mathrm{HA}$, the proportion of subjects with 4 -fold seroconversion was $75 \%$, the level of seroprotection was also $75 \%$, the antibody titer increase was $10.7-$ fold, and the geometric mean titer (GMT) of antibodies to A/H1N1pdm09 was 53.4; for the $7.5 \mu \mathrm{HA}$ dose, the proportion of 4 -fold seroconversion was $75 \%$, the antibody titer increase was 32.0 -fold, the GMT was 160.0 , and the level of seroprotection was $75 \%$. When administered at a higher dose (15 $\mu \mathrm{g} \mathrm{HA}$ ), the proportion of subjects with protective antibody titers increased from $75 \%$ to $83 \%$; however, the GMT and antibody titer increase were not significantly different $(P>0.05)$ to the group vaccinated at $7.5 \mu \mathrm{g} \mathrm{HA}$. Phase II clinical studies of the 3.75 and $7.5 \mu \mathrm{g}$ HA doses of Refluvac ${ }^{\circledR}$ vaccine should be performed in a larger cohort of healthy volunteers aged 18-60. The effective immunogenicity of low doses of Refluvac@ vaccine may enable increased production of pandemic influenza vaccines, and thus provide more people with a safe, effective vaccine.
\end{abstract}

Keywords: Influenza A (H1N1) pdm09; Whole-virion vaccine; Safety, immunogenicity

\section{Introduction}

In 2009, humankind encountered its first pandemic of the twentyfirst century in the form of a new variant of the influenza A (H1N1) pdm09 virus containing a triple reassortment of RNA segments from human, swine and avian influenza strains $[1,2]$. Unlike the regular seasonal influenza virus, the emerging pandemic virus was notable for its rapid spread throughout the population [1]. According to the World Health Organization (WHO), as of June 25, 2010, the influenza A (H1N1) pdm09 pandemic had affected 214 countries and caused 18,209 human deaths [3]. An accurate estimate of the total number of infected people could not be established because per WHO recommendations, screening for the influenza virus was canceled in July 2009. Such tests would have exceeded the capacity of laboratories even in most welldeveloped countries where the incidence rate was high.

Large-scale vaccination of populations is the most effective countermeasure for controlling pandemic outbreaks. However, due to numerous sequence differences, seasonal vaccines against influenza did not provide significant cross protection against the pandemic influenza A (H1N1) pdm09 virus [4,5]. Accordingly, a number of pharmaceutical companies, including Baxter (Czech Republic/ Austria) [6], GlaxoSmithKline (Belgium) [6,7], Novartis (Italy) [6,8], Omninvest (Hungary) [6,9], CSL Biotherapies (Australia) [10,11], Sanofi-Pasteur (France/USA) [12], and Hualan Biological Bacterium Company (China) [13-15] clinically tested monovalent inactivated pandemic influenza vaccines based on the same technologies they use to produce seasonal influenza vaccines. Preparation of these pandemic vaccines was based on the original influenza virus A/California/7/2009 (H1N1) pdm09 isolate. Most of the currently licensed monovalent pandemic vaccines are in the form of split-virion agents and do not contain adjuvant (except for the Pandemrix ${ }^{\mathrm{TM}}$ and Arepanrix ${ }^{\mathrm{TM}}$ [GlaxoSmithKline] vaccines which contain the oil adjuvant $\mathrm{ASO}_{\mathrm{A}}$ ). A smaller number of pandemic vaccines are presented in the form of whole-virions (Celvapan, Baxter; Fluval P, Omninvest) or subunit vaccines (Focetria, Novartis), and may or may not contain adjuvant (e.g. aluminum phosphate, MF-59). Clinical studies have shown that all three types of inactivated pandemic vaccine can induce a sufficient level of immune response complying with the Committee for Proprietary Medicinal Products (CPMP) and European Agency for the Evaluation of Medicinal Products (EMEA) requirements in volunteers of different age groups, mainly via single administration of vaccines containing 5.25-15.0 $\mu$ g hemagglutinin (HA) [6-15]. In a newer approach, a novel delta inulin adjuvanted recombinant HA-based pandemic vaccine was also shown to induce high levels of seroprotection against H1N1) pdm09 in human subjects, albeit requiring 11-45 $\mu \mathrm{g}$ of HA consistent with the lower immunogenicity of recombinant proteins when compared

*Corresponding author: Kaissar Tabynov, 080409, Republic of Kazakhstan Zhambulskaya oblast, Kordaiskiy rayon, Gvardeiskiy, Kazakhstan, Tel: + 7 (72636) 7-22-28; E-mail: tabynov_81@mail.ru

Received July 24, 2013; Accepted October 09, 2013; Published October 13, 2013

Citation: Tabynov K, Khairullin B, Kydyrbayev Z, Sandybayev N, Stukova M, et al. (2013) Preliminary Assessment of Safety and Immunogenicity of an Inactivated Whole-Virion Vaccine against Influenza A (H1N1) Pdm09 Containing Aluminum Hydroxide Adjuvant: A Randomized, Blinded Phase I Clinical Study. J Vaccines Vaccin 4: 205. doi: 10.4172/2157-7560.1000205

Copyright: (c) 2013 Tabynov K, et al. This is an open-access article distributed under the terms of the Creative Commons Attribution License, which permits unrestricted use, distribution, and reproduction in any medium, provided the original author and source are credited. 
Citation: Tabynov K, Khairullin B, Kydyrbayev Z, Sandybayev N, Stukova M, et al. (2013) Preliminary Assessment of Safety and Immunogenicity of an Inactivated Whole-Virion Vaccine against Influenza A (H1N1) Pdm09 Containing Aluminum Hydroxide Adjuvant: A Randomized, Blinded Phase I Clinical Study. J Vaccines Vaccin 4: 205. doi: 10.4172/2157-7560.1000205

to inactivated virus [16]. Notably, the lowest HA content required to induce a sufficient immune response was seen when whole-virion or adjuvant-containing vaccines were used. The HA content is the most important limiting factor when developing a pandemic vaccine, as existing production capacities for seasonal influenza vaccines are rather limited and are unable to meet the worldwide demand for a pandemic vaccine. Thus, a higher number of doses of vaccines with low antigenic loads can be manufactured.

On this basis, in order to create a pandemic vaccine to protect the population of Kazakhstan and neighbouring Central Asian countries, an inactivated whole-virion vaccine (Refluvac ${ }^{\circledR}$ ) against H1N1pdm09 containing aluminum hydroxide adjuvant was developed at the Research Institute for Biological Safety Problems (Kazakhstan). We previously demonstrated the safety and immunogenicity of this vaccine in preclinical studies conducted at research centers in Russia (The Research Institute of Influenza, Institute of Toxicology, Chemical and Pharmaceutical Academy) and Kazakhstan (The National Center for Expertise on Drugs) [17,18]. On the basis of these preclinical studies, Refluvac $^{\varpi}$ vaccine was recommended for a phase I clinical study of single intramuscular administration of $3.75,7.5$, and $15.0 \mu \mathrm{g}$ doses of $\mathrm{HA}$ in $0.5 \mathrm{ml}$ volume

Here, we describe the results of a randomized, blinded, dosedependent placebo-controlled phase I clinical study of single administration of Refluvac $c^{\circ}$, an inactivated whole-virion vaccine against H1N1pdm09 containing aluminum, with dose escalation in healthy human volunteers aged 18-60 years.

\section{Materials and Methods}

\section{Vaccine}

This clinical study used the inactivated whole-virion vaccine Refluvac $^{\varpi}$ against H1N1pdm09 containing alhydrogel adjuvant which was manufactured at the Research Institute for Biological Safety Problems. The vaccine is based on the reassortment strain NIBRG121xp (NIBSC code: 09/166), which was obtained from the National Institute for Biological Standards and Control (UK) where it was produced via reverse genetic engineering of the influenza strains $\mathrm{A} /$ California/7/2009 (H1N1) pdm09 and A/PR/8/34 (H1N1). This strain was provided to the Research Institute for Biological Safety Problems by the WHO for use in vaccine development. The Refluvac ${ }^{\circ}$ vaccine production procedure has been described in our previous study [18]. The compositions for the Refluvac ${ }^{\oplus}$ vaccine containing three different levels of HA are given in Table 1 . All vaccine specimens were packed in $2.5 \mathrm{ml}$ vials (5 doses in each).

\section{Study design}

A randomized, blinded placebo-controlled phase 1 clinical study of single administration with dose escalation was performed to assess the safety, tolerability, and immunogenicity of the inactivated wholevirion vaccine (Refluvac ${ }^{\oplus}$ ) containing aluminum adjuvant in healthy

\begin{tabular}{|c|c|c|c|}
\hline \multirow{2}{*}{ Vaccine composition per $\mathbf{0 . 5} \mathbf{~ m L}$} & \multicolumn{3}{|c|}{ Vaccine samples } \\
\cline { 2 - 4 } & $3.75 \mu \mathrm{g} \mathrm{HA}$ & $7.5 \mu \mathrm{g} \mathrm{HA}$ & $15 \mu \mathrm{g} \mathrm{HA}$ \\
\hline Hemagglutinin, $\mu \mathrm{g}$ & $3.75 \pm 0.5$ & $7.5 \pm 1.0$ & $15.0 \pm 2.0$ \\
\hline Aluminum hydroxide $\left(\mathrm{Al}^{3+}\right), \mathrm{mg}$ & $0.125 \pm 0.025$ & $0.25 \pm 0.05$ & $0.5 \pm 0.1$ \\
\hline Thimerosal, $\mu \mathrm{g}$ & $50 \pm 7$ & $50 \pm 7$ & $50 \pm 7$ \\
\hline PBS $^{*}, \mathrm{~mL}$ & up to $0.5 \mathrm{~mL}$ & up to $0.5 \mathrm{~mL}$ & up to $0.5 \mathrm{~mL}$ \\
\hline
\end{tabular}

*containing NaCl (Sigma, St. Louis, MO, USA) $8.7 \mathrm{~g} ; \mathrm{Na}_{2} \mathrm{HPO}_{4} .7 \mathrm{H}_{2} \mathrm{O}$ (Sigma) 1.88 g; $\mathrm{NaH}_{2} \mathrm{PO}_{4} \cdot 2 \mathrm{H}_{2} \mathrm{O}$ (Sigma) $0.46 \mathrm{~g}$; water to $1 \mathrm{~L}$.

Table 1: Composition of the Refluvac ${ }^{\circledR}$ vaccine samples. human volunteers aged 18-60 years. The study was performed at the clinic of the Research Institute of Influenza (Saint-Petersburg, Russia) from 17 June to 19 July 2010.

The volunteers were each vaccinated with one of three doses of $\operatorname{Refluvac}^{\circ}(3.75,7.5$, or $15.0 \mu \mathrm{g} \mathrm{HA})$, with 12 volunteers receiving each dose. The dose of Refluvac ${ }^{\circ}$ was increased based on the decisions of the Data Monitoring Committee according to the pre-specified study plan. The volunteers were randomized into vaccine and placebo groups in a 2:1 ratio respectively. Randomization of volunteers was carried out by sealed envelopes.

The study procedure is shown in Table 2. Either the vaccine or placebo (sterile water for injection) was administered as a single intramuscular $0.5 \mathrm{ml}$ dose into the deltoid area of the volunteer's non-dominant arm on Day 0 of the study. Active monitoring of the volunteers was conducted for 7 days after the vaccination (the day of vaccination and the 6 days after). Subsequently, starting on the evening of Day 7 through to Day 21 of the study (study completion), the volunteers kept Self-Observation Diaries where they registered the occurrence of any symptoms. The study only included volunteers who had $\leq 1: 10$ antibody titers to A/H1N1pdm09 in the hemagglutinin inhibition assay (HIA), and who were seronegative to HIV (CombiBest anti-HIV-1+2; VECTOR-BEST, Novosibirsk, Russia), hepatitis B (Best anti-HCV; VECTOR-BEST), and hepatitis C (Vector B-HBsantigen; VECTOR-BEST), in the enzyme-linked immunosorbent assay (ELISA). Screening studies were performed within 2 weeks before the start of the vaccination.

Each volunteer participated in the study for a total of no more than 21 days. The study included a screening stage, a baseline visit, visits 2-7, and a final visit 8 on Day 21. The interval between the screening visit and the baseline visit was no more than 14 days, and 21 days between the baseline visit and final visit.

Before the study started and any study procedures were performed, the Study Protocol, Volunteer Information including an informed consent form, and other information for the volunteers were reviewed and approved by Ethics Committee of the Federal Service on Surveillance in Health Care and Social Development (Ministry of Health and Social Development of the Russian Federation) and the Local Ethics Committee of the Research Institute of Influenza (St. Petersburg, Russia).

The study was conducted in compliance with Good Clinical Practice in the Russian Federation (Order of the Ministry of Healthcare of the Russian Federation No. 266 as of 19.06.2003), the Declaration of Helsinki which was adopted by the XVII session of the World Health Assembly (1964) and reviewed during the World Health Assembly session in 2000, as well as national legal requirements.

The clinical study protocol BPK-I-00-01/2010 was performed based on clinical study authorization No. 269 of June, 102010 issued by the Ministry of Public Health and Social Development of the Russian Federation of Federal Service on Surveillance in Health Care and Social Development.

\section{Evaluation of the reactogenicity and safety of the vaccine}

The reactogenicity and safety of the vaccine were evaluated in all of the volunteers participating in the study according to the following parameters: the intensity and relationship of all local and systemic reactions occurring within 7 days (the day of vaccination and the 6 days 
Citation: Tabynov K, Khairullin B, Kydyrbayev Z, Sandybayev N, Stukova M, et al. (2013) Preliminary Assessment of Safety and Immunogenicity of an Inactivated Whole-Virion Vaccine against Influenza A (H1N1) Pdm09 Containing Aluminum Hydroxide Adjuvant: A Randomized, Blinded Phase I Clinical Study. J Vaccines Vaccin 4: 205. doi: 10.4172/2157-7560.1000205

Page 3 of 6

\begin{tabular}{|l|c|c|c|c|c|c|c|}
\hline \multicolumn{1}{|c|}{ Procedure/Observation } & Screening & \multicolumn{3}{c|}{ Days after vaccination } & \multicolumn{2}{c|}{ Day 21 $\mathbf{2}$} \\
days
\end{tabular}

aBefore vaccination. ${ }^{\mathrm{b}} \mathrm{At} 20$ minutes and 2 hours after vaccination.

Table 2: Regulation of the phase I clinical study of the Refluvac ${ }^{\circledR}$ vaccine.

following) to the vaccination; the intensity and relationship of local and systemic reactions registered by the study participants from Day 8 through Day 21 of the study to the vaccination (based on the volunteers' records in the Self-Observation Diaries); the findings of neurological examinations; and laboratory analysis of specimens taken on Days 0,7 , and 21 (complete blood count and blood chemistry panel [C-reactive protein, alkaline phosphatase, glucose, creatinine, potassium, sodium, calcium, total bilirubin, alanine aminotransferase (ALT), aspartate transaminase (AST), total protein], and clinical urinalysis).

The intensity of symptoms were measured using four-point scales as follows: Local reactions: 0 , None (absence of symptoms); 1, Mild (hyperemia up to $50 \mathrm{~mm}$ in diameter or infiltration up to $25 \mathrm{~mm}$ in diameter); 2, Moderate (hyperemia more than $50 \mathrm{~mm}$ in diameter or infiltration up to $26-50 \mathrm{~mm}$ in diameter); and 3, Severe (infiltration more than $50 \mathrm{~mm}$ in diameter). Systemic reactions: 0 , None (absence of symptoms); 1, Mild (presence of slight symptoms); 2, Moderate (symptoms notably influence normal daily activities); and 3, Severe (symptoms impede normal daily activities). Fever: 0 , None $\left(\leq 37^{\circ} \mathrm{C}\right) ; 1$, Mild $\left(>37^{\circ} \mathrm{C}-<37.5^{\circ} \mathrm{C}\right) ; 2$, Moderate $\left(>37.6^{\circ} \mathrm{C}-<38.5^{\circ} \mathrm{C}\right)$; and 3 , Severe $\left(\geq 38.6^{\circ} \mathrm{C}\right)$.

An adverse event (AE) was regarded as any unfavorable or unexpected sign (including laboratory abnormalities), symptom, or disease (newly diagnosed or aggravated) time-related to administration of the vaccine. AE observed by the investigators (Days 1-7) were recorded in a case report form (CRF), and all $\mathrm{AE}$ which occurred after administration of the vaccine and recorded by the participants in their Self-Observation Diaries (Days 8-21) were transferred by the investigator into the CRF. All AE were imported into the study participant's CRF regardless of their intensity or causal relation to the vaccination.

\section{Evaluation of immune response}

The immune response to administration of Refluvac ${ }^{\bullet}$ vaccine was evaluated using a HAI assay [19] using chicken red blood cells. To remove non-specific inhibitors, blood test samples were treated with the receptor-destroying enzyme from Vibrio cholerae (Denka Seiken Co. Ltd., Japan). The geometric mean titer (GMT) of antibodies to A/H1N1pdm09 (on Day 0 [before vaccination] and 21); the seroconversion factor (on Day 21); the level of seroconversion (on Days 0 [before vaccination] and 21); and the level of seroprotection (on Days 0 [before vaccination] and 21) were determined.

\section{Statistical analyses}

Analysis of local and systemic adverse reactions was performed based on their degree of intensity. Consolidated figures for the occurrence of local and systemic reactions within 7 days after the vaccination for a single volunteer were analyzed. The evaluation was performed by assessing the maximal intensity of the adverse reactions observed within 7 days of the vaccination. The rates for the different groups of vaccinated individuals were compared with respect to the presence of adverse reactions within 7 days of each vaccination. AE were analyzed using the following parameters: number of volunteers having any AE; presence of drug-related AE; presence of Serious Adverse Events (SAE); presence of SAE related to other medicines taken; and presence of $\mathrm{AE}$ which led to withdrawal from the study. The occurrence of AE was defined as possibly, probably, or unlikely to be related to the vaccine; in cases where no information was available to categorize the relationship of the $\mathrm{AE}$ to the vaccine, the $\mathrm{AE}$ was regarded as inter-related to the vaccination.

Descriptive statistics show the geometric mean titer for $\mathrm{HI}$ of all groups at all visits. Results for HI titer below detection limit reported as ' $<10$ ' were set to 5 for further calculation. Where appropriate, $95 \%$ confidence intervals (CIs) were computed using the Wilson scorebased method.

\section{Results}

\section{Study volunteers}

The study was performed in 54 healthy volunteers of both sexes aged 18-60 years including 15 (27.8\%) males and 39 (72.2\%) females. Based on their demographic characteristics, the volunteers were distributed as evenly as possible into three groups for each dose level of the vaccine or the placebo group. The mean age of the volunteers in the group immunized with the lowest dose of the vaccine $(3.75 \mu \mathrm{g} \mathrm{HA})$ was slightly higher but not significantly different (45.8 years; range, 33-56 years), to that of the groups immunized with the $7.5 \mu \mathrm{g}$ HA dose (36.3 
Citation: Tabynov K, Khairullin B, Kydyrbayev Z, Sandybayev N, Stukova M, et al. (2013) Preliminary Assessment of Safety and Immunogenicity of an Inactivated Whole-Virion Vaccine against Influenza A (H1N1) Pdm09 Containing Aluminum Hydroxide Adjuvant: A Randomized, Blinded Phase I Clinical Study. J Vaccines Vaccin 4: 205. doi: 10.4172/2157-7560.1000205

years; range, 20-57 years) and $15 \mu \mathrm{g}$ HA dose (37.5 years; range 23 - 54 years) or the placebo group (39.5 years; range, 22 - 60 years).

\section{Safety assessment}

To assess the safety of the vaccine, we analyzed AE based on the results of laboratory and clinical tests (vital signs including blood pressure, heart rate, temperature; ECG examination; hematology panel, blood chemistry panel, and clinical urinalysis), physical and neurological examinations, and the volunteers' medical history.

A total of five AE were noted during the study in 5 of the 54 volunteers (9.26\%); four $\mathrm{AE}(7.4 \%)$ were considered to be probably related to administration of the vaccine, one $\mathrm{AE}(1.85 \%)$ was considered unlikely to be related to administration of the vaccine. The drug-related $\mathrm{AE}$ included three local reactions of mild intensity and one systemic reaction of moderate intensity. No AE of serious intensity were observed. None of the AE required additional therapy, and no volunteer was excluded from the study due to AE. No SAE were observed during the study (Table 3).

Within 20 minutes and 2 hours of vaccination, none of the 54 volunteers noted any AE (either local or systemic reactions). Six hours after the vaccination, three volunteers who had been administered the $7.5 \mu \mathrm{g}$ HA dose of Refluvac ${ }^{\varpi}$ vaccine noted mild "discomfort" at the injection site; this "discomfort" persisted until the next morning in one volunteer (Day 2 after the vaccination); the "discomfort" at the vaccine injection site was short-term in the other two cases. No local reactions in the form of hyperemia or infiltration were observed at the injection site in any other volunteers. One $\mathrm{AE}$ in the form of a short-term (less than 2 hours) increase in temperature to $37.8^{\circ} \mathrm{C}$ was noted 6 hours after vaccination in one volunteer who had been administered the $15 \mu \mathrm{g} \mathrm{HA}$ dose of Refluvac ${ }^{\bullet}$ vaccine.

No influence of the vaccination was observed in the results of the complete blood counts, blood chemistry panels, clinical urinalysis, or ECG examinations. After the vaccination, all of these parameters remained at the baseline level (data not shown).

\section{Assessment of vaccine immunogenicity}

Assessment of the immunogenicity of the vaccine in the setting of single administration was performed 21 days after the vaccination using the HAI assay. In the group of volunteers immunized with the $3.75 \mu \mathrm{g}$ HA dose of Refluvac ${ }^{\oplus}$ vaccine, the proportion of subjects with 4 -fold seroconversions was $75 \%$, the level of seroprotection was also $75 \%$, the increase in the antibody titer was 10.7 -fold, and the GMT of antibodies to influenza virus $\mathrm{A} / \mathrm{H} 1 \mathrm{~N} 1 \mathrm{pdm} 09$ was 53.4 ; in the group of volunteers immunized with the $7.5 \mu \mathrm{g}$ HA dose of Refluvac ${ }^{\bullet}$ vaccine, the proportion of subjects with 4 -fold seroconversions was $75 \%$, the increase in the antibody titer was 32.0-fold, the GMT was 160.0, and the level of seroprotection was $75 \%$.

In the group of volunteers immunized with the high dose (15 $\mu \mathrm{g}$ HA), the proportion of subjects with protective antibody titers increased from $75 \%$ (for the $7.5 \mu \mathrm{g}$ HA dose) to $83 \%$; however, no significant $(\mathrm{P}=0.02)$ increases in the GMT or antibody titer increase rate were observed in the volunteers immunized with the $15 \mu \mathrm{g}$ HA dose compared to those vaccinated with the $7.5 \mu \mathrm{g}$ HA dose of Refluvac ${ }^{\oplus}$. The HAI values for the three doses of Refluvac ${ }^{\circ}$ vaccine are shown in Table 4.

\section{Discussion}

The aim of this work was to perform a phase I clinical study to provide preliminary assessment of the safety, tolerability, and immunogenicity of the monovalent inactivated whole-virion vaccine Refluvac $^{\circ}$ containing aluminum adjuvant against the pandemic H1N1pdm09 virus in healthy volunteers aged 18-60 years. This research followed on from large-scale preclinical studies of Refluvac ${ }^{\oplus}$ performed at the research centers of the Russian Federation and Republic of

\begin{tabular}{|c|c|c|c|c|c|c|c|c|c|c|}
\hline \multirow[b]{2}{*}{ Number of volunteers } & \multicolumn{2}{|c|}{ Vaccine $3.75 \mu \mathrm{g}$ HA } & \multicolumn{2}{|c|}{ Vaccine $7.5 \mu \mathrm{g} H A$} & \multicolumn{2}{|c|}{ Vaccine $15 \mu \mathrm{g} H A$} & \multicolumn{2}{|c|}{ Placebo } & \multicolumn{2}{|c|}{ Total } \\
\hline & $\begin{array}{l}\text { Absolute } \\
\text { number }\end{array}$ & $\%$ & $\begin{array}{l}\text { Absolute } \\
\text { number }\end{array}$ & $\%$ & $\begin{array}{l}\text { Absolute } \\
\text { number }\end{array}$ & $\%$ & $\begin{array}{l}\text { Absolute } \\
\text { number }\end{array}$ & $\%$ & $\begin{array}{c}\text { Absolute } \\
\text { number }\end{array}$ & $\%$ \\
\hline Number of volunteers & 12 & $\begin{array}{c}22.2(13.2- \\
34.9)\end{array}$ & 12 & $\begin{array}{c}22.2(13.2- \\
34.9)\end{array}$ & 12 & $\begin{array}{l}22.2(13.2- \\
34.9)\end{array}$ & 18 & $\begin{array}{l}33.3(22.2- \\
46.6)\end{array}$ & 54 & $\begin{array}{c}100.0 \\
(93.4-100)\end{array}$ \\
\hline Number with at least $1 \mathrm{AE}$ & 1 & $\begin{array}{c}8.3 \\
(1.5-35.4)\end{array}$ & 3 & $\begin{array}{c}25.0 \\
(8.9-53.2)\end{array}$ & 1 & $\begin{array}{c}8.3 \\
(1.5-35.4)\end{array}$ & 0 & 0 & 5 & $\begin{array}{c}9.26 \\
(4-19.9)\end{array}$ \\
\hline $\begin{array}{l}\text { Number with at least one } \\
\text { vaccination-related AE }\end{array}$ & 0 & 0 & $3^{*}$ & $\begin{array}{c}25.0 \\
(8.9-53.2)\end{array}$ & $1^{* *}$ & $\begin{array}{c}8.3 \\
(1.5-35.4)\end{array}$ & 0 & 0 & 4 & $\begin{array}{c}7.4 \\
(2.9-17.6)\end{array}$ \\
\hline Number with no $A E$ & 11 & $\begin{array}{c}91.6 \\
(64.6-98.5)\end{array}$ & 9 & $\begin{array}{c}75.0 \\
(46.8-91.1)\end{array}$ & 11 & $\begin{array}{c}91.6 \\
(64.6-98.5)\end{array}$ & 18 & $\begin{array}{c}100.0 \\
(82.4-100)\end{array}$ & 49 & $\begin{array}{c}90.7 \\
(80.1-96)\end{array}$ \\
\hline $\begin{array}{l}\text { Number with at least one serious } \\
\text { adverse drug reaction }\end{array}$ & 0 & 0 & 0 & 0 & 0 & 0 & 0 & 0 & 0 & 0 \\
\hline
\end{tabular}

*Local reactions of mild intensity occurring within 6 hours of vaccination; **Systemic reactions of moderate intensity occurring within 6 hours of vaccination. Table 3: Adverse events to Refluvac $₫$ vaccine observed during the study.

\begin{tabular}{|c|c|c|c|c|c|c|c|c|}
\hline \multirow{3}{*}{$\begin{array}{l}\text { Agent } \\
\text { ee } 3.75 \mu \mathrm{gA}\end{array}$} & \multirow{3}{*}{$\begin{array}{c}\text { Number of } \\
\text { individuals }\end{array}$} & \multirow{2}{*}{\multicolumn{2}{|c|}{$\begin{array}{c}\text { Number of individuals with 4-fold } \\
\text { seroconversions } \\
\text { No. }(\%) \text { of Vol. }(95 \% \mathrm{Cl})^{* *}\end{array}$}} & \multicolumn{2}{|c|}{ GMT of antibodies* $(95 \% \mathrm{Cl})$} & \multirow{3}{*}{$\begin{array}{c}\text { Antibody titer } \\
\text { increase rate }\end{array}$} & \multirow{2}{*}{\multicolumn{2}{|c|}{$\begin{array}{c}\text { Number of individuals with } \\
\text { protective antibody titers }(>1: 40) \\
\text { No. }(\%) \text { of Vol. }(95 \% \mathrm{Cl})^{\star *}\end{array}$}} \\
\hline & & & & \multirow{2}{*}{$\begin{array}{c}\text { Before } \\
\text { vaccination } \\
5.0(5.0-5.0)\end{array}$} & \multirow{2}{*}{\begin{tabular}{|c|}
$\begin{array}{c}\text { Day } 21 \text { after } \\
\text { vaccination }\end{array}$ \\
$53.3(22.3-127.4)$
\end{tabular}} & & & \\
\hline & & 10 & $75.0(46.8-91.1)$ & & & & 9 & $75.0(46.8-91.1)$ \\
\hline Vaccine $7.5 \mu \mathrm{g} \mathrm{HA}$ & 12 & 12 & $75.0(46.8-91.1)$ & $5.0(5.0-5.0)$ & $160.0(61.4-416.8)$ & $32.0(12.2-83.3)$ & 9 & $75.0(46.8-91.1)$ \\
\hline Vaccine $15 \mu \mathrm{g} \mathrm{HA}$ & 12 & 12 & $83.3(55.2-95.3)$ & $5.9(4.5-7.8)$ & $126.9(59.4-271.3)$ & $21.3(10.4-43.6)$ & 10 & $83.3(55.2-95.3)$ \\
\hline Placebo & 18 & 0 & $0.00(0.0-17.6)$ & $5.83(4.8-7.0)$ & $6.3(4.9-7.9)$ & $1.0(0.9-1.2)$ & 0 & $0.0(0.0-17.6)$ \\
\hline
\end{tabular}

${ }^{*} \mathrm{GMT}$ of antibodies against pandemic influenza A/H1N1pdm09

${ }^{*}{ }^{*} 95 \%$ Cls were computed using the Wilson score-based method 

an Inactivated Whole-Virion Vaccine against Influenza A (H1N1) Pdm09 Containing Aluminum Hydroxide Adjuvant: A Randomized, Blinded Phase I Clinical Study. J Vaccines Vaccin 4: 205. doi: 10.4172/2157-7560.1000205

Kazakhstan which demonstrated the safety and high immunogenicity of the vaccine in laboratory animals $[17,18]$.

Development of the inactivated, whole-virion adjuvanted (aluminum hydroxide) vaccine Refluvac $^{\bullet}$ against pandemic influenza A (H1N1) pdm09 in Kazakhstan was based on reports [20-24] which demonstrated that whole-virion vaccines, as compared with split and subunit types, induce a higher degree of immunogenicity in both laboratory animal models and humans during clinical trials. According to Kistner et al. [25], the higher immunogenicity of whole-virion vaccines compared to split vaccines is due to their ability to induce both Th1 and Th2 immune responses. Moreover, the immunity conferred by inactivated whole-virion vaccines shows cross-protection, i.e. the vaccines are able to protect against genetic variants of the pandemic influenza virus $[21,23]$. However, in general whole-virion influenza vaccines are recognized to be more reactogenic and pyrogenic than split and subunit equivalents, limiting their use for seasonal influenza vaccines.

Aluminum hydroxide adjuvant was added to the Refluvac ${ }^{\circledast}$ vaccine composition with the aim of increasing immunogenicity and allowing use of the lowest antigen load possible, which in turn may decrease the reactogenicity of the whole-virion vaccine. Aluminum hydroxide is frequently used as an adjuvant in clinical practice. The adjuvant action of aluminum hydroxide was long attributed to its ability to adsorb antigens onto its surface, thus increasing the release time and therefore the duration of contact between an antigen and antigen-presenting cells. However, it was subsequently shown that adsorbed antigens are released within first few hours after the injection [26]. It has been shown that aluminum adjutants induce strong innate immune responses that consist of an influx of neutrophils, eosinophils, NK cells, CD11b+ monocytes and dendritic cells (DCs) to the site of injection [27-30]. The cellular signaling pathways triggered by aluminum that activate DCs and macrophages and direct toward Th2 immune responses and effective humoral immunity against antigens have only recently been addressed. Mice genetically lacking both MyD88 and TRIF, adaptor molecules of the TLR signaling pathway, still respond to alum adjuvant by mounting robust antigen specific antibody responses [31,32] with alum's adjuvant activity related to inflammasome activation and to release of DNA from phagocytic cells killed by alum that in tuen activates innate immune receptors [33]. Negative findings in recent clinical studies of whole-virion vaccine against influenza $\mathrm{A} / \mathrm{H} 5 \mathrm{~N} 1$ formulated with aluminum adjuvants have called into question the immunostimulating action of alum adjuvant for influenza vaccines [24]. However, on the basis of previous studies [17,18,34,35] which clearly demonstrated the adjuvant action of aluminum hydroxide when formulated with the Refluvac ${ }^{\diamond}$ vaccine, we again used alumcontaining vaccine for this clinical study, although in the absence of a whole-virion alone arm without adjuvant, we cannot know whether the alum adjuvant played any enhancing role or not in our vaccine. Another argument for including aluminum hydroxide in the vaccine composition is its stabilizing effect for unstable antigens [36] although in general HA antigens are relatively stable.

This phase I clinical study of the monovalent inactivated wholevirion vaccine Refluvac ${ }^{\oplus}$ against pandemic influenza A (H1N1) pdm09 demonstrated that single administration of the vaccine at a dose of 3.75 , 7.5, or $15.0 \mu \mathrm{g}$ HA to a small number of human subjects aged 18-60 years did not identify any safety issues, as no severe AE or SAE related to the vaccination were observed during the study. The $\mathrm{AE}$ occurring in the volunteers immunized with Refluvac ${ }^{\circledR}$ were mainly mild local reactions (7.5 $\mu \mathrm{g} \mathrm{HA}, 25 \%)$; only one case of a moderate systemic reaction was observed (15.0 $\mu \mathrm{g} \mathrm{HA}, 8.3 \%)$. No vaccination-related AE were observed in the volunteers immunized with the $3.75 \mu \mathrm{g}$ HA dose of Refluvac ${ }^{\circ}$ vaccine. No abnormalities compared to baseline were observed in the clinico-laboratory analyses of the volunteers on Days 7 and 21 after the vaccination, irrespective of the dose of vaccine administered. We compared the results obtained in this study with data from phase I-II clinical studies of a whole-virion vaccine against influenza $\mathrm{A} / \mathrm{H} 5 \mathrm{~N} 1$ (Baxter) containing aluminum adjuvant, which used the wild-type virus A/Vietnam/1203/2004 generated in Vero cell cultures [24] as a vaccine strain; and a whole-virion vaccine against pandemic influenza A/H1N1pdm09 containing aluminum phosphate adjuvant (Fluval $\mathrm{P}$, Omninvest, Hungary), which used the reassortant virus NYMC X-179A (New York Medical College) generated in chicken embryos as a vaccine strain [9]. We regard the above-mentioned vaccines as the closest analogs of Refluvac ${ }^{\circ}$. Comparison of the data revealed the Refluvac $^{\oplus}$ vaccine to be equally reactogenic as its closest analog Fluval P vaccine (6.0 $\mu$ g HA: local reactions $11.2 \%$, systemic reactions $5.1 \%$ ) [9], and slightly superior to the vaccine produced by Baxter $(3.75 \mu \mathrm{g}$ HA: local reactions $29 \%$, systemic reactions $51 \%$; $7.5 \mu \mathrm{HA}$ : local reactions $22 \%$, systemic reactions $31 \% ; 15.0 \mu \mathrm{g}$ HA: local reactions $28 \%$, systemic reactions $30 \%$ ) [24]. Indeed, based on data from clinical studies, of all types of monovalent inactivated pandemic influenza vaccines, the most reactogenic vaccines were those containing squalene oil emulsions including MF-59,(Novartis, Marburg, Germany; 3.75-7.5 $\mu \mathrm{g}$ HA: local reactions $80.0 \%$, systemic reactions $40.0 \%$ ) [8] or ASO3A, (GlaxoSmithKline, Dresden, Germany; $5.25 \mu \mathrm{g}$ HA: local reactions $88.9 \%$, systemic reactions $41.3 \%$ ) [7].

The immunogenicity of the Refluvac ${ }^{\circledast}$ vaccine was analyzed by determining the seroconversion rate, seroprotection rate, antibody titer increase rate, and GMT. This study demonstrated that all three doses of the vaccine were immunogenic in adults aged 18-60 years in the setting of a single intramuscular administration. In the group of volunteers immunized with the $3.75 \mu \mathrm{g}$ HA dose of the Refluvac ${ }^{\circ}$ vaccine, the proportion of subjects with 4 -fold seroconversions was $75 \%$, the level of seroprotection was also $75 \%$, the increase in the antibody titer was 10.7 fold, and the GMT of antibodies to A/H1N1pdm09 was 53.4. Compared to the $3.75 \mu \mathrm{g}$ HA group, most indices of vaccine immunogenicity were higher in the group of volunteers immunized with the $7.5 \mu \mathrm{g}$ HA dose of the Refluvac ${ }^{\circ}$ vaccine: the increase in antibody titer was 32.0 -fold, and the GMT was 160.0; however, the level of seroconversions (75\%) and seroprotection $(75 \%)$ was similar to that of the volunteers immunized with the $3.75 \mu \mathrm{g}$ HA dose. When the vaccine was administered at a higher dose (15 $\mu \mathrm{g} \mathrm{HA})$, the proportion of subjects with protective antibody titers increased from $75 \%$ to $83 \%$; however, the GMT and antibody titer increase rate observed in this group were not significantly different to that of the volunteers vaccinated with the $7.5 \mu \mathrm{g}$ HA dose of Refluvac ${ }^{\circ}$. Our findings demonstrate that single administration of the Refluvac $^{\bullet}$ vaccine at a dose of $3.75 \mu \mathrm{g}, 7.5 \mu \mathrm{g}$, or $15 \mu \mathrm{g}$ HA has marked immunogenic activity against A/H1N1pdm09 influenza virus, with all of the studied values and parameters complying with the European requirements of the CPMP EMEA for inactivated influenza vaccines. Notably, the Refluvac ${ }^{\oplus}$ vaccine was immunologically effective in adult volunteers after single administration at a dose of $3.75 \mu \mathrm{g} \mathrm{HA}$, which is 2-4 times lower than the immunogenic doses of other whole-virion split or subunit vaccines against pandemic influenza A/H1N1pdm09 [6-15]. The effective immunogenicity of low doses of the Refluvac ${ }^{\oplus}$ vaccine may enable increased production of pandemic influenza vaccines from the current limited production capacities, and thus provide more people with a safe and effective vaccine.

The results of this phase I study allow us to recommend the 
Citation: Tabynov K, Khairullin B, Kydyrbayev Z, Sandybayev N, Stukova M, et al. (2013) Preliminary Assessment of Safety and Immunogenicity of an Inactivated Whole-Virion Vaccine against Influenza A (H1N1) Pdm09 Containing Aluminum Hydroxide Adjuvant: A Randomized, Blinded Phase I Clinical Study. J Vaccines Vaccin 4: 205. doi: 10.4172/2157-7560.1000205

performance of phase II clinical trials of the Refluvac ${ }^{\circ}$ vaccine at doses of $3.75 \mu \mathrm{g} \mathrm{HA}$ and $7.5 \mu \mathrm{g} \mathrm{HA}$ in a larger cohort of healthy volunteers aged 18-60 years.

\section{Acknowledgment}

The authors would like to express their gratitude to employees of the Research Institute for Biological Safety Problems, K.T. Sultankulova, O.V. Chervyakova and E. Tailakova for their assistance in the preparation of vaccines for clinical trials; M.M. Kassenov, Y.N. Volgin for their assistance in the quality control of vaccines. The work was carried out under the research program "Monitoring, study and development of diagnostic, preventive and therapy methods for A/ H1N1 influenza" for 2009-2011 funded by the Science Committee of the Ministry of Education and Science of the Republic of Kazakhstan.

\section{References}

1. Novel Swine-Origin Influenza A (H1N1) Virus Investigation Team, Dawood FS, Jain S, Finelli L, Shaw MW, et al. (2009) Emergence of a novel swine-origin influenza A (H1N1) virus in humans. N Engl J Med 360: 2605-2615

2. Zimmer SM, Burke DS (2009) Historical perspective--Emergence of influenza $A$ (H1N1) viruses. N Engl J Med 361: 279-285.

3. WHO (2010) Pandemic (H1N1) 2009: update 106.

4. Centers for Disease Control and Prevention (CDC) (2009) Serum cross-reactive antibody response to a novel influenza $A(\mathrm{H} 1 \mathrm{~N} 1)$ virus after vaccination with seasonal influenza vaccine. MMWR Morb Mortal Wkly Rep 58: 521-524.

5. Pascua PN, Song MS, Lee JH, Park KJ, Kwon HI, et al. (2009) Evaluation of the efficacy and cross-protectivity of recent human and swine vaccines against the pandemic (H1N1) 2009 virus infection. PLoS One 4: e8431.

6. Johansen K, Nicoll A, Ciancio BC, Kramarz P (2009) Pandemic influenza $A(H 1 N 1) 2009$ vaccines in the European Union. Euro Surveill 14: 19361.

7. Roman F, Vaman T, Gerlach B, Markendorf A, Gillard P, et al. (2010) Immunogenicity and safety in adults of one dose of influenza A H1N1v 2009 vaccine formulated with and without AS03A-adjuvant: preliminary report of an observer-blind, randomised trial. Vaccine 28: 1740-1745.

8. Clark TW, Pareek M, Hoschler K, Dillon H, Nicholson KG, et al. (2009) Trial of 2009 influenza A (H1N1) monovalent MF59-adjuvanted vaccine. N Engl J Med 361: $2424-2435$

9. Vajo Z, Tamas F, Sinka L, Jankovics I (2010) Safety and immunogenicity of a 2009 pandemic influenza A H1N1 vaccine when administered alone or simultaneously with the seasonal influenza vaccine for the 2009-10 influenza season: a multicentre, randomised controlled trial. Lancet 375: 49-55.

10. Greenberg ME, Lai MH, Hartel GF, Wichems CH, Gittleson C, et al. (2009) Response to a monovalent 2009 influenza A (H1N1) vaccine. N Engl J Med 361: $2405-2413$

11. Nolan T, McVernon J, Skeljo M, Richmond P, Wadia U, et al. (2010) Immunogenicity of a monovalent 2009 influenza $A(\mathrm{H} 1 \mathrm{~N} 1)$ vaccine in infants and children: a randomized trial. JAMA 303: 37-46.

12. Plennevaux E, Sheldon E, Blatter M, Reeves-Hoché MK, Denis M (2010) Immune response after a single vaccination against 2009 influenza A H1N1 in USA: a preliminary report of two randomised controlled phase 2 trials. Lancet 375: 41-48.

13. Zhu FC, Wang H, Fang HH, Yang JG, Lin XJ, et al. (2009) A novel influenza A $(\mathrm{H} 1 \mathrm{~N} 1)$ vaccine in various age groups. N Engl J Med 361: 2414-2423.

14. Liang XF, Li L, Liu DW, Li KL, Wu WD, et al. (2011) Safety of influenza A (H1N1) vaccine in postmarketing surveillance in China. N Engl J Med 364: 638-647.

15. Liang XF, Wang HQ, Wang JZ, Fang HH, Wu J, et al. (2010) Safety and immunogenicity of 2009 pandemic influenza A H1N1 vaccines in China: a multicentre, double-blind, randomised, placebo-controlled trial. Lancet 375: 56-66.

16. Gordon DL, Sajkov D, Woodman RJ, Honda-Okubo Y, Cox MM, et al. (2012) Randomized clinical trial of immunogenicity and safety of a recombinant H1N1/2009 pandemic influenza vaccine containing Advaxâ, $\notin$ polysaccharide adjuvant. Vaccine 30: 5407-5416.
17. Mamadalyiev S, Nurpeysova A, Khairullin B, Kydyrbaev Zh, Kassenov M, et al (2011) Preclinical Testing of Refluvac $\circledast$, A vaccine against pandemic influenza A/H1N1v. J Appl Environ Biol Sci 1: 48-53.

18. Tabynov K, Kydyrbayev Z, Sansyzbay A, Khairullin B, Ryskeldinova S, et al. (2012) Immunogenic and protective properties of the first Kazakhstan vaccine against pandemic influenza A (H1N1) pdm09 in ferrets. Virol Sin 27: 345-352.

19. WHO (2002) WHO manual on animal influenza diagnosis and surveillance. Geneva.

20. Lin J, Zhang J, Dong X, Fang H, Chen J, et al. (2006) Safety and immunogenicity of an inactivated adjuvanted whole-virion influenza A (H5N1) vaccine: a phase I randomised controlled trial. Lancet 368: 991-997.

21. Ninomiya A, Imai M, Tashiro $M$, Odagiri $T$ (2007) Inactivated influenza H5N1 whole-virus vaccine with aluminum adjuvant induces homologous and heterologous protective immunities against lethal challenge with highly pathogenic H5N1 avian influenza viruses in a mouse model. Vaccine 25 3554-3560.

22. Subbarao K, Luke C (2007) H5N1 viruses and vaccines. PLoS Pathog 3: e40.

23. Vajo Z, Kosa L, Visontay I, Jankovics M, Jankovics I (2007) Inactivated whole virus influenza A (H5N1) vaccine. Emerg Infect Dis 13: 807-808.

24. Ehrlich HJ, Müller M, Oh HM, Tambyah PA, Joukhadar C, et al. (2008) A clinical trial of a whole-virus H5N1 vaccine derived from cell culture. N Engl J Med 358 2573-2584.

25. Kistner O, Crowe BA, Wodal W, Kerschbaum A, Savidis-Dacho H, et al. (2010) A whole virus pandemic influenza H1N1 vaccine is highly immunogenic and protective in active immunization and passive protection mouse models. PLoS One 5: e9349.

26. Gupta RK, Chang AC, Griffin P, Rivera R, Siber GR (1996) In vivo distribution of radioactivity in mice after injection of biodegradable polymer microspheres containing 14C-labeled tetanus toxoid. Vaccine 14: 1412-1416.

27. Calabro S, Tortoli M, Baudner BC, Pacitto A, Cortese M, et al. (2011) Vaccine adjuvants alum and MF59 induce rapid recruitment of neutrophils and monocytes that participate in antigen transport to draining lymph nodes. Vaccine 29: 1812-1823.

28. Kool M, Soullié T, van Nimwegen M, Willart MA, Muskens F, et al. (2008) Alum adjuvant boosts adaptive immunity by inducing uric acid and activating inflammatory dendritic cells. J Exp Med 205: 869-882.

29. McKee AS, Munks MW, MacLeod MK, Fleenor CJ, Van Rooijen N, et al. (2009) Alum induces innate immune responses through macrophage and mast cel sensors, but these sensors are not required for alum to act as an adjuvant for specific immunity. J Immunol 183: 4403-4414.

30. Mosca F, Tritto E, Muzzi A, Monaci E, Bagnoli F, et al. (2008) Molecular and cellular signatures of human vaccine adjuvants. Proc Natl Acad Sci U S A 105 10501-10506.

31. Gavin AL, Hoebe K, Duong B, Ota T, Martin C, et al. (2006) Adjuvant-enhanced antibody responses in the absence of toll-like receptor signaling. Science 314 1936-1938.

32. Palm NW, Medzhitov R (2009) Immunostimulatory activity of haptenated proteins. Proc Natl Acad Sci U S A 106: 4782-4787.

33. Marichal T, Ohata K, Bedoret D, Mesnil C, Sabatel C, et al. (2011) DNA released from dying host cells mediates aluminum adjuvant activity. Nat Med 17: $996-1002$

34. Sansyzbay AR, Erofeeva MK, Khairullin BM, Sandybayev NT, Kydyrbayev ZK, et al. (2013) An inactivated, adjuvanted whole virion clade 2.2 H5N1 (A Chicken/Astana/6/05) influenza vaccine is safe and immunogenic in a single dose in humans. Clin Vaccine Immunol 20: 1314-1319.

35. Nakayama T, Kumagai T, Ishii KJ, Ihara T (2012) Alum-adjuvanted H5N1 whole virion inactivated vaccine (WIV) induced lgG1 and IgG4 antibody responses in young children. Vaccine 30: 7662-7666.

36. Rappuoli R, Bagnoli F (2011) Vaccine Design: Innovative Approaches and Novel Strategies. Caister Academic Press. 